\title{
Efficient prediction of siRNAs with siRNArules 1.0: An open-source JAVA approach to siRNA algorithms
}

\author{
TORGEIR HOLEN \\ Centre for Molecular Biology and Neuroscience (CMBN), University of Oslo, Oslo, Norway
}

\begin{abstract}
RNAi interference and siRNA have become useful tools for investigation of gene function. However, the discovery that not all siRNA are equally efficient made necessary screens or design algorithms to obtain high activity siRNA candidates. Several algorithms have been published, but they remain inefficient, obscure, or commercially restricted. This article describes an opensource JAVA program that is surprisingly efficient at predicting active siRNAs (Pearson correlation coefficient $r=0.52, n=526$ siRNAs). Furthermore, this version 1.0 sets the stage for further improvement of the free code by the open-source community (http://sourceforge.net/).
\end{abstract}

Keywords: RNAi; siRNA; design; algorithm; bioinformatics

\section{INTRODUCTION}

The genomic immune system of RNA interference was first convincingly described by Fire et al. (1998) in the roundworm Caenorhabditis elegans, where double-stranded RNA was used to induce depletion of RNA with sequence similarity. At first mammalian cells seemed incapable of mounting an RNAi response to dsRNA. Then Elbashir et al. (2001a) and Caplen et al. (2001) found a way of inducing RNAi using 21-mer RNA duplexes, short interfering RNA (siRNA). This seemed to pave the way for this useful tool also in human cells. However, it was quickly demonstrated that not all siRNAs are active (Holen et al. 2002), setting the stage for the present problem: how to identify the most active siRNA candidates.

The first clue to the underlying rules was that a very small shift in the siRNA's position could ruin the activity (Holen et al. 2002). A mere 6-nucleotide (nt) shift caused a highly active siRNA to lose almost all potency, indicating strong local sequence dependency. Early attempts at determining rules, e.g., an average GC content in the siRNA itself, met with failure (Holen et al. 2002; Kawasaki et al. 2003), as small-scale public attempts at screens showed no strong correlation with GC content.

Meanwhile, commercial companies invested in large screens of siRNAs and announced that they had found patterns incorporated into algorithms (that were available for a fee). Several public online services are also available

Reprint requests to: Torgeir Holen, CMBN, University of Oslo, Oslo, Norway; e-mail: torgeir.holen@medisin.uio.no; fax: +47-0-22851488.

Article published online ahead of print. Article and publication date are at http://www.rnajournal.org/cgi/doi/10.1261/rna.81006.
(Henschel et al. 2004; Naito et al. 2004; Ren et al. 2006). However, the publication of the underlying data sets for the private sector screens have been patchy (Khvorova et al. 2003) and the published algorithms not very effective (Saetrom and Snove 2004). No large academic screens have been performed, indicating that the knowledge of existence of commercial sets that could be published at any time have, in fact, delayed the progress of the RNAi field in this regard.

Now, with the important advent of the publication of the large Novartis data set (Huesken et al. 2005) and online software (http://www.biopredsi.org/start.html), and the collection of several smaller sets by Saetrom and Snove (2004), the stage is set for a pooling of minds in an open-source effort. This work presents the kernel of an algorithm that simply exploits the readily apparent overrepresentation of certain nucleotides in certain positions of the siRNA.

This siRNA design program, siRNArules 1.0, is written in the very accessible JAVA programming language and can be demonstrated to be surprisingly efficient, both on published sets and on hitherto unpublished (or partly published) screens performed in our laboratory. More importantly, this first version of the software could be easily improved upon and fiddled with, in private, by anyone with the simplest of JAVA compilers, making possible a collective open-source effort to produce ever more efficient, freely available siRNA design software.

\section{METHODS}

The code of siRNArules 1.0 one has three parts, all of which are comprehensibly annotated (see supplemental file 1, 
siRNArules.java). First, the program reads an input file (mRNA_input.dat) containing the mRNA sequence of interest and converts it into a list of all possible siRNAs (technically siRNA JAVA objects in an array of such siRNA objects). All numbers, spaces, or signs other than A, C, G, $\mathrm{T}$, and $\mathrm{U}$ (and their lowercase counterparts a, c, g, t, and $\mathrm{u}$ ) are ignored. This means the program can read any DNA or RNA file format as long as there is no additional text containing nucleotide letters.

The JAVA code used for siRNArules 1.0 has been kept simple to the point of simplicity to ensure that anybody should be able to fiddle with the code (see Supplemental File 1, siRNArules.java).

Each siRNA object is evaluated by the core algorithm of the program in a simple list of if-then statements. In this first version, the algorithm is a comparison, to a list of known nucleotide biases, for of each of the 21 nucleotide positions of the antisense strand. Finally, the list of siRNA objects is then sorted and written to a result file (output.dat). Improving the core algorithm will be the main task of the open-source community.

The scoring table for the algorithm is summarized in Table 1. Briefly, the list of nucleotide biases in each position consists of a positive or a negative score for each position, based mainly on the percentage overrepresentation or underrepresentation of these nucleotides in large siRNA reference sets, such as the Novartis study (Huesken et al. 2005) and other studies as summarized by (Saetrom and Snove 2004).

In position 10 , counted from the $5^{\prime}$ end of the antisense strand, for example, the nucleotide A is $54 \%$ overrepre- sented in the Novartis set. The siRNArules 1.0 algorithm thus adds a score of 54 points to each siRNA object that has an $\mathrm{A}$ in position 10. The choice of a simple arithmetic sum, which aims to identify the siRNAs in an mRNA that are the most similar to the best siRNAs and most dissimilar to the worst siRNA, might seem simplistic. The reason for this choice is due to intrinsic paradoxes in the reference sets.

For examples of such paradoxes, consider the set of the best 200 siRNAs and the set of the worst 200 siRNAs in the large Novartis screen (Huesken et al. 2005). The two sets are not quite consistent. Since, for example, A is strongly overrepresented in position 10 in the set of the 200 best siRNA, one could expect A to be underrepresented in position 10 in the set of the 200 worst siRNA. So it is, but only weakly at $11 \%$. The scoring table for the algorithm is therefore at present a compromise between these conflicting rules.

Several other positions are more or less consistent between the two sets, but there are large paradoxes also, for example at position 19, where A is very strongly overrepresented in the set of the worst 200 siRNA, at $80 \%$ the strongest bias seen in the entire set. Yet in position 19 in the set of 200 best siRNA no negative bias is found for $\mathrm{A}$ at all. More examples of paradoxical positions are positions $3,4,5,11,13$, and 18, all of which have nucleotides $>30 \%$ overrepresented in one set but with no corresponding bias in the other.

One mechanical explanation for this type of paradox is that the dominating hypothesis for siRNA activity, the biased strand-loading hypothesis proposed by Zamore and colleagues (Schwarz et al. 2003), is probably incomplete.

TABLE 1. Algorithm scoring table for siRNArules 1.0

\begin{tabular}{|c|c|c|}
\hline mRNA position & Positive rules (from set of best siRNAs) & Negative rules (from set of worst siRNAs) \\
\hline 1 & $A=66, C=-18, G=-31, U=78$ & $A=-16, C=59, G=80, U=-32$ \\
\hline 2 & $A=20, G=-12, U=40$ & $A=-17, C=53, G=13, U=-16$ \\
\hline 3 & $A=48, C=-8, G=-9, U=2$ & \\
\hline 4 & & $C=20, G=34, U=-11$ \\
\hline 5 & $U=41$ & $A=15, C=15$ \\
\hline 6 & $A=18, U=18$ & $A=29$ \\
\hline 7 & $A=26, C=-13, G=-9, U=38$ & $A=-10, C=52, G=12, U=-11$ \\
\hline \multicolumn{3}{|r|}{ 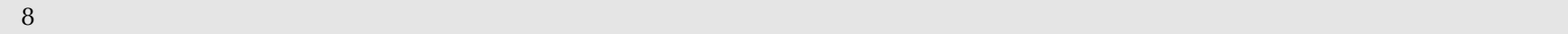 } \\
\hline \multicolumn{3}{|l|}{9} \\
\hline 10 & $A=54, G=-22, U=-10$ & $\mathrm{~A}=-11, \mathrm{G}=8, \mathrm{U}=24$ \\
\hline 11 & $A=10, U=29$ & $A=-8, C=38, G=22, U=-13$ \\
\hline \multicolumn{3}{|r|}{ 管 } \\
\hline 13 & & $A=-10, C=20, G=36$ \\
\hline 14 & $A=22, G=-12, U=22$ & $C=1, G=27$ \\
\hline \multicolumn{3}{|c|}{ ( } \\
\hline \multicolumn{3}{|l|}{16} \\
\hline \multicolumn{3}{|l|}{17} \\
\hline 18 & & $A=37$ \\
\hline 19 & $C=31, G=1$ & $A=80, C=-14, G=-11, U=-6$ \\
\hline 20 & $A=16, U=16$ & $C=28, G=2$ \\
\hline 21 & $A=16, C=-15, G=28$ & $A=13, C=31, G=-9$ \\
\hline
\end{tabular}


Recently our group has demonstrated another mechanism for siRNA inactivation, whereby the RISC enzyme restoration after cleavage is slowed down by high binding energy in the siRNA:mRNA duplex $3^{\prime}$ of the cleavage point in the mRNA (S.E. Moe, J.G. Sørbø, and T. Holen, unpubl.).

This siRNA inactivation mechanism is probably one underlying cause of the overrepresentation of A and underrepresentation of $\mathrm{G}$ in position 10 in the set of good siRNAs. Such a two-tier mechanism for siRNA activity (strand loading into RISC and RISC enzyme restoration) can have opposite requirements for high activity and end up making contradictory rules.

Therefore siRNArules 1.0 operates with two scoring sums: one for the Positive Rules deduced from the set of the best siRNAs and one for the Negative Rules deduced from the set of the worst siRNAs. The rank is calculated by a simple sum of these two values. How these rules should be weighted to obtain the most efficient algorithm is a challenge for the open-source community.

Another main goal of the open-source community is to improve on the scoring table (Table 1) to obtain clues about what parts of the siRNA are most important.

Novartis reported using sophisticated statistical software, so-called neural networks, a statistical procedure for machine "learning," to optimize their algorithm BIOPREDsi (Huesken et al. 2005). They report having obtained a Pearson correlation coefficient of $r=0.66$ on an independent test set (with $r=0.67$ for the training set). Furthermore, they report having obtained Pearson coefficients of $0.57,0.45$, and 0.55 for the published sets of (Vickers et al. 2003), (Harborth et al. 2003), and (Reynolds et al. 2004).

Despite reporting the best predictions in the public siRNA field so far, such "black box" approaches to algorithms have one drawback. One cannot easily inspect the final weighted nodes of the trained algorithm to obtain information comprehensible to human minds. Neither can one fiddle with the algorithm based upon mechanical insights, such as the RISC enzymatic restoration mechanism mentioned above.

The first version of siRNArules is in fact rather efficient (see Table 2 for comparisions on 18 published and four unpublished test sets, and the discussion below). With its rather simplistic approach to statistics, it obtains a Pearson correlation coefficient of 0.55 for the set of all 526 siRNAs. For the published sets also tested by Novartis (Huesken et al. 2005), (Vickers et al. 2003), (Harborth et al. 2003), and (Reynolds et al. 2004), the Pearson correlations were $0.42,0.33$, and 0.55 , respectively, something that is almost comparable to the Novartis' BIOPREDsi predictions and better than most other algorithms published and available, using the Amarzguioui algorithm and the Saetrom and Snove study as a benchmark (Supplemental Table 1; Amarzguioui and Prydz 2004; Saetrom and Snove 2004). Also using ROC sensitivity versus specificity plots, siRNA rules 1.0 was shown to have the highest prediction power (Supplemental Table 1).

Thus siRNArules 1.0 already has a surprisingly high efficiency and with much scope for improvement in the algorithm by the open-source community (http://sourceforge.net/).

\section{RESULTS AND DISCUSSION}

To test siRNArules 1.0's predictions, the (Saetrom and Snove 2004) collection of various published sets of siRNA results was used and a range of different measures of success calculated (Table 2). As the results in the literature are very heterogeneous, a standardization procedure was introduced, where the functionality score of each siRNA depended on relative score compared within its own set.

For example, in the study by Harborth et al. (2003), almost all the siRNAs were reported to have a depletion efficiency of $80 \%$ or better, while none of the siRNAs in the study by Vickers et al. (2003) were reported to be better than $75 \%$. One interpretation of the difference in overall siRNA efficacy between these two studies is that mRNA targets might differ in intrinsic target accessibility.

In our own practice we have seen the tendency to saturation results, that is, at $12 \mathrm{~h}$ after transfection of siRNA, two siRNAs might have different efficacy, but at $24 \mathrm{~h}$, both have depleted the mRNA down to saturation levels. Thus some targets might even be too soft, as opposed to hard targets where little siRNA silencing is seen at all, if the siRNA measurements are taken at an inappropriate time. As a consequence of this, siRNArules 1.0 and this study do not use an siRNA's efficacy per se, but the relative rank in relation to other possible siRNAs to the same mRNA target.

In order to be able to compare different data sets, the sets were ordered with regard to efficiency and two sets defined from the median, a best half and a worse half, the simplest of all possible statistical tests. In the larger data sets, the two sets of the best quartile and the worst quartile was also identified, in order to see if these more stringent conditions gave better predictions. Conversely, all possible siRNAs from each mRNA were evaluated by siRNArules 1.0, and the predictions of specific siRNAs were scored after rank of efficiency in the full set of all possible siRNAs for each mRNA.

Another problem with earlier siRNA studies and algorithms is that they have generally considered the target sequence of the siRNA to be $19 \mathrm{nt}$. This bias was introduced originally by Tuschl et al. (Elbashir et al. 2001b,c), as the $3^{\prime}$ overhang of the antisense strand was concluded not to participate as strongly in the target recognition. The Novartis study found that position 20 and position 21 indeed do influence the siRNA efficiency (Huesken et al. 2005), but their study is somewhat weakened by using deoxyribonucleotides, and not ribonucleotides, in the two terminal nucleotides. In a small, early study, our group 


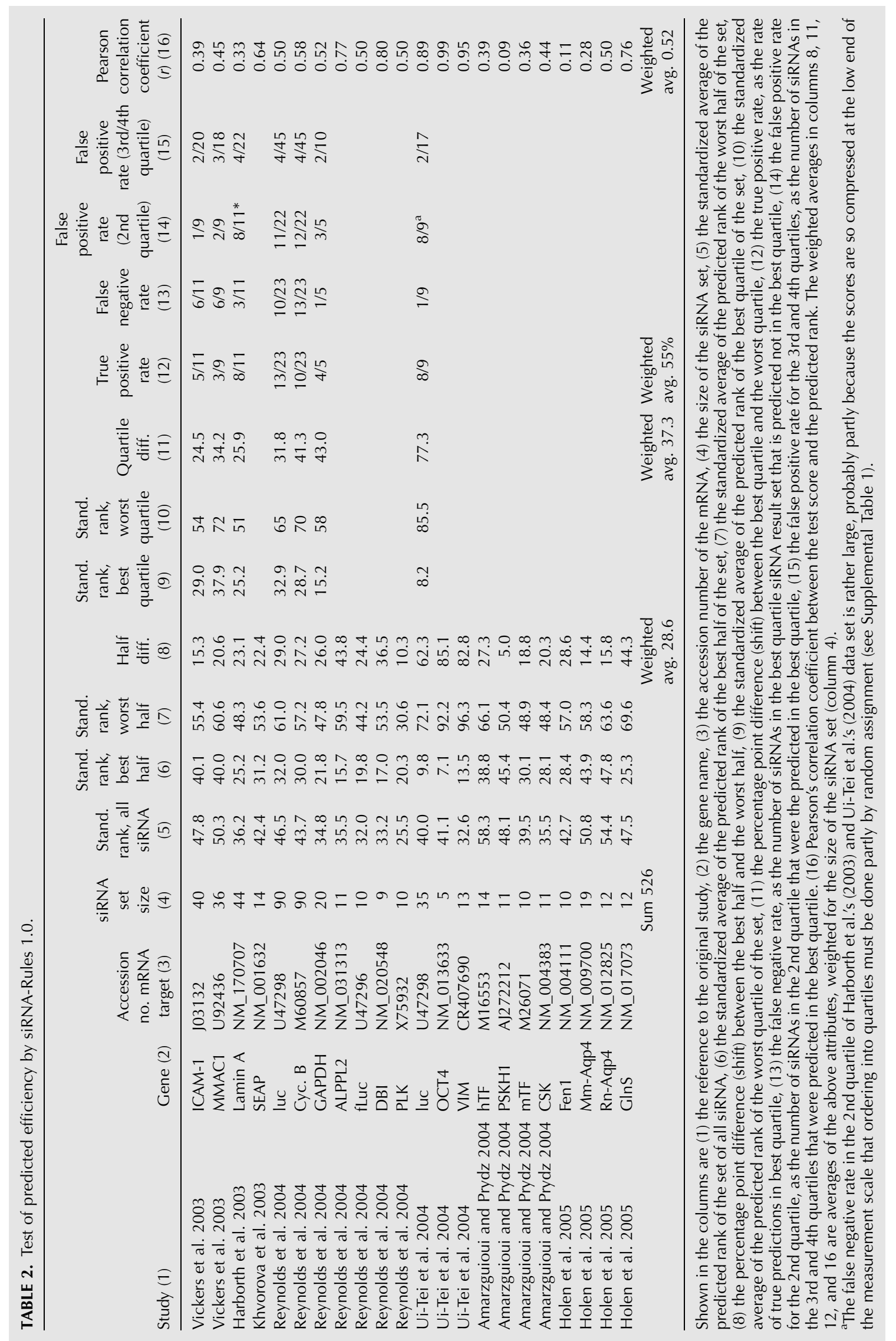


found no difference between DNA and RNA nucleotides in the terminal, overhanging nucleotides (Holen et al. 2002). Therefore siRNArules 1.0 uses 21-mer recognition (Table 1).

Yet another consideration is that the data sets used for validation are rather small, making reliable statistics difficult. Also, as we shall see, the siRNAs chosen for the studies have obviously not been random, as, except for the very first studies, the siRNA sample itself seems to be biased with respect to the predictions of siRNArules 1.0, and, quite probably, biased with respect to the real siRNA efficacy.

The validation of siRNArules 1.0 considered 18 different published data sets. In addition four unpublished (or partially published) sets from our own practice were considered. Each siRNA in the sets (in total 526 siRNAs) was individually identified and converted from 19-mer to 21-mer if necessary. Other sets are available in online databases, but most of these are from very small sets (Ren et al. 2006).

The predictions, with a PositiveValue and NegativeValue, were sorted by the sum of these. As discussed above, this choice is a compromise, as the two reference sets contain paradoxes. siRNArules 1.0 prints both the PositiveValue and the NegativeValue score, so that the users can decide whether they would want to aim for the siRNA most similar to the best siRNA set or to aim for avoiding the similarity to the set of the worst siRNAs. Whether these scores give better prediction has not been tested, as in the following discussion the sorted rank is the final indicator of predicted siRNA efficacy.

The results from the predictions are tabulated in Table 2. In all 22 sets, siRNArules 1.0 worked better than would be expected by random. The best half had a better predicted rank than the worst half in all cases (Table 2, columns 6,7). As a quantitative measure of the prediction, the percentage point difference (shift) in predicted rank from the best set to the worst set was calculated (Table 2, column 8).

When comparing the best quartile to the worst quartile, the siRNArules 1.0 's predictions were measurably better. Only seven of the 18 sets had 20 siRNAs or more and were considered large enough for comparisons of quartiles.

The theoretical maximum shift for comparisons of halves, assuming a completely homogenous distribution of siRNAs over the $100 \%$ range and a perfect prediction, is a 50-percentage-point shift, and a 75-percentage-point shift for comparison of best and worst quartiles. From the table it can be seen that the shift in comparison of halves averaged 28.6 percentage points and the shift in quartiles, 37.3 percentage points. Thus there is room for improvement in siRNArules 1.0's predictions, but by this simplest of statistical measures, the program's predictions are rather good.

In fact, there are reasons to believe that the predictions are not even as good as this weighted average. First, some of the data sets have shifts that are absurdly large, as in the case of the Ui-Tei et al. (2004) sets. Upon inspection of these rather small sets, it can be seen that the distribution is skewed both ways, toward the very good and the very bad siRNAs, thus making a shift possible larger than the theoretical maximum dependent on homogenous distribution of the siRNA set.

Furthermore, as a random data set would score a predicted rank around the 50th percentile, one can see that several of the siRNA data sets are not random. Except for a few early sets published in 2002, the average rank of the siRNAs sets deviate much from 50\% (Table 2, column 5), probably as a result of the influence of publications by (Schwarz et al. 2003) and (Khvorova et al. 2003), in Cell in 2003, and in the continual refinements of the prediction tools by these research groups themselves.

The early publications, such as (Vickers et al. 2003) and Amarzguioui's $h T F$ gene (Holen et al. 2002), show a distribution of the prediction close to the 50\% mark, with a progression in the later sets (Amarzguioui and Prydz 2004). At the other extreme, one of the sets from Dharmacon (Reynolds et al. 2004) is predicted to be distributed around the 25th percentile, presumably a set late in the process of making efficient algorithms at this company.

A distribution shift might not be very intuitively useful for a biologist, so I also computed the true positive rate (Table 2, column 12), the false negative rate (column 13), and false positive rate (columns 14 and 15) for the first, the second, and the combined third and fourth quartiles, respectively. Thus siRNArules, on average, for the largest data sets, manages to predict a $55 \%$ true positive rate for the required best quartile. This means, for example, that one has a $91 \%$ chance of obtaining a good siRNA (in the best quartile) if one tests three different siRNAs against a target. It should be noted that the predicted siRNAs might also affect other mRNA targets. Users should carefully consider running seven-word BLAST at NCBI, as recommended previously (Snove and Holen 2004).

Finally, a Pearson correlation coefficient was calculated for all sets, giving a weighted average of 0.52 (Table 2, column 16), which is almost comparable to the 0.65 score of Novartis BIOPREDsi (Huesken et al. 2005). It should be noted that a Pearson correlation coefficient can be mathematically proven to be the most effective only when the two variables contain a Gaussian distribution (although it should be a useful test also over other distributions), and since siRNArules 1.0's score is predicted rank (by definition a uniform distribution), thus the comparison of Pearson scores with Novartis' BIOPREDsi are not unproblematic. ROC sensitivity versus specificity plots, demonstrating rather high predictive power, have been made for the six largest sets, for the aggregate total set (526 siRNA), and for an independent aggregate set excluding all siRNA from our own laboratory and those used in design of the Amarzguioui algorithm (427 siRNA) (Supplemental Table 1). For even more sophisticated statistical tests see (Saetrom and Snove 
2004), and (Baldi et al. 2000). However, overly complicated statistics might even serve to obfuscate, as the main problem in siRNA studies is the lack of good quality data sets.

In conclusion, there is large variation in the prediction efficacy, from the simplest to the most advanced statistical measures, between the different sets. Some of this can be attributed to random error in small sets; some can be attributed to mistakes by siRNArules 1.0 or even the measurements in the original studies. However, some of this variation might point to hitherto unrecognized insights in siRNA efficacy and might lead to new versions of siRNArules from the open-source community (http:// sourceforge.net/).

\section{SUPPLEMENTAL MATERIAL}

Supplemental Table 1 is available at http://folk.uio.no/torgeirh/ siRNArules/siRNArules_Supplemental-Table-1.xls. Supplemental File 1 is available at http://folk.uio.no/torgeirh/ siRNArules.java.

\section{ACKNOWLEDGMENTS}

T.H. has a grant from the Norwegian Cancer Society. I am grateful to Stig Hagen Dommarsnes, who contributed with advice resulting in shorter and more beautiful JAVA code, and to members of the Ottersen laboratory for continual support and encouragement.

Received March 8, 2006; accepted June 12, 2006.

\section{REFERENCES}

Amarzguioui, M. and Prydz, H. 2004. Algorithm for selection of functional siRNA sequences. Biochem. Biophys. Res. Commun. 316: 1050-1058.

Baldi, P., Brunak, S., Chauvin, Y., Andersen, C.A.F., and Nielsen, H. 2000. Assessing the accuracy of prediction algorithms for classification: An overview. Bioinformatics 16: 412-424.

Caplen, N.J., Parrish, S., Imani, F., Fire, A., and Morgan, R.A. 2001. Specific inhibition of gene expression by small double-stranded RNAs in invertebrate and vertebrate systems. Proc. Natl. Acad. Sci. 98: 9742-9747.

Elbashir, S.M., Harborth, J., Lendeckel, W., Yalcin, A., Weber, K., and Tuschl, T. 2001a. Duplexes of 21-nucleotide RNAs mediate RNA interference in cultured mammalian cells. Nature 411: 494-498.

Elbashir, S.M., Lendeckel, W., and Tuschl, T. 2001b. RNA interference is mediated by 21- and 22-nucleotide RNAs. Genes \& Dev. 15: 188-200.

Elbashir, S.M., Martinez, J., Patkaniowska, A., Lendeckel, W., and Tuschl, T. 2001c. Functional anatomy of siRNAs for mediating efficient RNAi in Drosophila melanogaster embryo lysate. EMBO J. 20: 6877-6888.

Fire, A., Xu, S., Montgomery, M.K., Kostas, S.A., Driver, S.E., and Mello, C.C. 1998. Potent and specific genetic interference by double-stranded RNA in Caenorhabditis elegans. Nature 391: 806-811.

Harborth, J., Elbashir, S.M., Vandenburgh, K., Manninga, H., Scaringe, S.A., Weber, K., and Tuschl, T. 2003. Sequence, chemical, and structural variation of small interfering RNAs and short hairpin RNAs and the effect on mammalian gene silencing. Antisense Nucleic Acid Drug Dev. 13: 83-105.

Henschel, A., Buchholz, F., and Habermann, B. 2004. DEQOR: A web-based tool for the design and quality control of siRNAs. Nucleic Acids Res. 32: W113-W120.

Holen, T., Amarzguioui, M., Wiiger, M.T., Babaie, E., and Prydz, H. 2002. Positional effects of short interfering RNAs targeting the human coagulation trigger Tissue Factor. Nucleic Acids Res. 30: $1757-1766$.

Holen, T., Moe, S.E., Sørbø, J.G., Meza, T.J., Ottersen, O.P., and Klungland, A. 2005. Tolerated wobble mutation in siRNAs decrease specificity, but can enhance activity in vivo. Nucleic Acids Res. 33: 4704-4710.

Huesken, D., Lange, J., Mickanin, C., Weiler, J., Asselbergs, F., Warner, J., Meloon, B., Engel, S., Rosenberg, A., Cohen, D., et al. 2005. Design of a genome-wide siRNA library using an artificial neural network. Nat. Biotechnol. 23: 995-1001.

Kawasaki, H., Suyama, E., Iyo, M., and Taira, K. 2003. siRNAs generated by recombinant human Dicer induce specific and significant but target site-independent gene silencing in human cells. Nucleic Acids Res. 31: 981-987.

Khvorova, A., Reynolds, A., and Jayasena, S.D. 2003. Functional siRNAs and miRNAs exhibit strand bias. Cell 115: 209-216.

Naito, Y., Yamada, T., Ui-Tei, K., Morishita, S., and Saigo, K. 2004. siDirect: Highly effective, target-specific siRNA design software for mammalian RNA interference. Nucleic Acids Res. 32: W124W129.

Ren, Y., Gong, W., Xu, Q., Zheng, X., Lin, D., Wang, Y., and Li, T. 2006. siRecords: An extensive database of mammalian siRNAs with efficacy ratings. Bioinformatics 22: 1027-1028.

Reynolds, A., Leake, D., Boese, Q., Scaringe, S., Marshall, W.S., and Khvorova, A. 2004. Rational siRNA design for RNA interference. Nat. Biotechnol. 22: 326-330.

Saetrom, P. and Snove Jr., O. 2004. A comparison of siRNA efficacy predictors. Biochem. Biophys. Res. Commun. 321: 247-253.

Schwarz, D.S., Hutvagner, G., Du, T., Xu, Z., Aronin, N., and Zamore, P.D. 2003. Asymmetry in the assembly of the RNAi enzyme complex. Cell 115: 199-208.

Snove Jr., O. and Holen, T. 2004. Many commonly used siRNAs risk off-target activity. Biochem. Biophys. Res. Commun. 319: 256263.

Ui-Tei, K., Naito, Y., Takahashi, F., Haraguchi, T., Ohki-Hamazaki, H., Juni, A., Ueda, R., and Saigo, K. 2004. Guidelines for the selection of highly effective siRNA sequences for mammalian and chick RNA interference. Nucleic Acids Res. 32: 936-948.

Vickers, T.A., Koo, S., Bennett, C.F., Crooke, S.T., Dean, N.M., and Baker, B.F. 2003. Efficient reduction of target RNAs by small interfering RNA and RNase H-dependent antisense agents. A comparative analysis. J. Biol. Chem. 278: 7108-7118. 

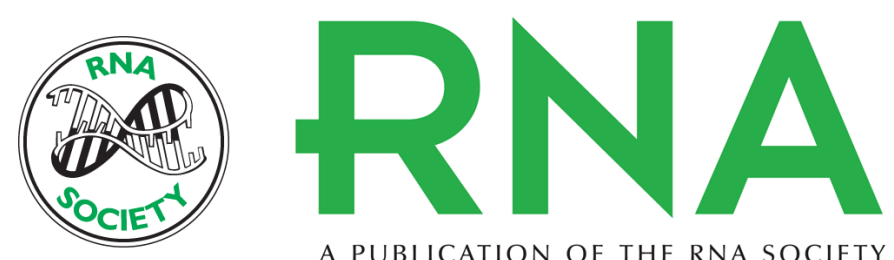

A PUBLICATION OF THE RNA SOCIETY

\section{Efficient prediction of siRNAs with siRNArules 1.0: An open-source JAVA approach to siRNA algorithms}

Torgeir Holen

RNA 2006 12: 1620-1625

References This article cites 22 articles, 4 of which can be accessed free at:

http://rnajournal.cshlp.org/content/12/9/1620.full.html\#ref-list-1

License

Email Alerting Receive free email alerts when new articles cite this article - sign up in the box at the Service top right corner of the article or click here.

To subscribe to RNA go to:

http://rnajournal.cshlp.org/subscriptions 